\title{
Aportes de la psicología positiva a la comprensión y el abordaje de los comportamientos suicidas
}

\author{
Aixa L. Galarza ${ }^{1}$
}

\section{Artículo}

Material original autorizado para la publicación en la revista Psicodebate. Facultad de Ciencias Sociales. Universidad de Palermo.

Recibido 16-10-2015 | Aceptado 20-11-2015

\section{Resumen}

Tradicionalmente, el estudio y abordaje psicológico de los comportamientos suicidas se abocó a la identificación de los factores de riesgo asociados. Sin embargo, progresivamente en las últimas décadas y en consonancia con los desarrollos llamados salugénicos, la investigación en el tema ha ido virando hacia la identificación y comprensión de los factores protectores o resilientes, entendidos como aquellos que actúan como amortiguadores ante el desarrollo de suicidalidad. Se entiende por enfoque salugénico al conjunto de desarrollos orientados a investigar las fortalezas y recursos personales que promueven un mayor potencial de salud en las personas y dan cuenta de las diferencias individuales para afrontar las adversidades. Dentro de este enfoque, resulta particularmente interesante la psicología positiva en tanto ha sido el movimiento que logró la consolidación de estos desarrollos que iniciara a mediados del siglo XX. En este marco, el trabajo que se presenta se propone revisar los aportes de la psicología positiva a la conceptualización, evaluación y tratamiento de los comportamientos suicidas. Se exponen básicamente tres aportes: optimismo y suicidalidad, la teoría de las emociones positivas, y la perspectiva de la teoría de la autodeterminación en relación con la teoría interpersonal del suicido. Se discuten las implicaciones.

Palabras Clave: comportamientos suicidas, enfoque salugénico, psicología positiva, optimismo, emociones positivas, teoría de la autodeterminación.

1 Consejo Nacional de Investigaciones Científicas y Técnicas (CONICET), Grupo de Investigación en Evaluación Psicológica GIEPsi-IPSIBAT, Facultad de Psicología, Universidad Nacional de Mar del Plata - Argentina; aixa_galarza@live.com.ar 


\title{
Contributions of positive psychology to the understanding and approach of and suicidal behaviors
}

\begin{abstract}
Traditionally, the psychological study and approach of suicidal behaviors aimed to the identification of the associated risk factors. However, progressively in recent decades and in line with developments called salutogenic, research on the subject has been turning to the identification and understanding of protective and resilient factors, referring as those that act as buffers against suicidality. By salutogenic approach it is understood the body of theoretical developments oriented to study personal strengths and resources that promote health potential on people and allow to detect individual differences in coping with adversity. Within this approach, it is particularly interesting the positive psychology, so it has been the movement that consolidate these developments, which initiate at mid twentieth century. In this context, the work presented aimed to review the contributions of positive psychology to the conceptualization, assessment and treatment of suicidal behaviors. Basically, they are presented three contributions: optimism and suicidality, theory of positive emotions, and the perspective of the theory of self-determination in relation to the interpersonal theory of suicide. The implications are discussed.
\end{abstract}

Keywords: suicidal behaviors, salutogenic approach, positive psychology, optimism, positive emotions, self-determination theory. 
Desde los años '40 y, sobre todo, a partir de la década del '60, se observa una tendencia creciente de desarrollos teóricos y empíricos orientados a investigar las fortalezas y recursos personales que promueven un mayor potencial de salud en las personas, y llevan a consolidar un nuevo enfoque denominado salugénico (e.g. Allport, 1937; Antonovsky, 1988; Bandura, 1977; Buhgental, 1964; Maslow, 1954; May, 1940, 1953; Rogers, 1972; Rutter, 1987). El punto de convergencia es el estudio de los aspectos positivos o saludables de las personas que dan cuenta de las diferencias individuales para afrontar las adversidades y sobreponerse a ellas de un modo adaptativo. ${ }^{\mathrm{i}}$

El desarrollo más reciente y que ha favorecido la consolidación del enfoque en la actualidad es la llamada psicología positiva (Seligman \& Csikszentmyhalyi, 2000), definida como el estudio científico de las fortalezas y emociones positivas de las personas y de las instituciones que favorecen su promoción. En la última década este enfoque se ha consolidado de manera progresiva tanto a nivel internacional como en Argentina (Casullo, 2008). Ha aumentado considerablemente el número de investigaciones, publicaciones y reuniones científicas, el desarrollo de instrumentos y la aplicación en los ámbitos profesionales de la psicología.

Específicamente, este trabajo se propone indagar en los aportes de la psicología positiva a una problemática concreta: los comportamientos suicidas ${ }^{\mathrm{ii}}$ en lo que se refiere a conceptualización, evaluación y tratamiento. Para ello, primero se realizará una breve exposición histórica y conceptual del enfoque.

\section{Antecedentes. De la psicopatología al bienestar}

Desde sus comienzos, la psicología ha estado orientada hacia una perspectiva psicopatológica basada en el modelo médico y por ende centrada en el déficit, el malestar y en el alivio de los síntomas. Sin embargo, a mediados del siglo XX, como contrapartida al psicoanálisis y al conductismo, comenzaron a surgir en Estados Unidos lineamientos teóricos e investigaciones con una perspectiva más positiva acerca del ser humano que se conoce como tercera fuerza y tiene sus raíces en el humanismo y el existencialismo (Mariñelarena-Dondena \& Gancedo, 2011). Con el paso del tiempo, estos desarrollos han comenzado a ser agrupados bajo la denominación de enfoque salugénico. Entre sus antecedentes se encuentran aportes de diferentes escuelas psicológicas, pero sin duda entre los más destacados están la psicología humanista y la teoría de la motivación humana de Maslow (1954), quien podría señalarse como el portavoz de esta corriente, al plantear la necesidad de estudiar las capacidades del "hombre sano" e introducir conceptos como los de necesidades de desarrollo y autoactualización. Siguiendo a Prada (2005), otros autores cuyos trabajos han sido clave son Allport (1965), quien describió la personalidad madura; Rotter 
(1966) con su conceptualización del locus de control; Rogers (1972) a través de la idea de funcionamiento óptimo de la personalidad; Bandura (1977) con el concepto de autoeficacia; y las conceptualizaciones de Rutter sobre resiliencia (1987).

Particularmente relevante es el modelo salugénico de Antonovsky (1988). El autor planteó el concepto de orientación salugénica y la concepción de la salud enfermedad como extremos de un continuo (Gancedo, 2008; Mariñelarena-Dondena \& Gancedo, 2011). Esta propuesta no solo enfatiza la importancia de reducir la probabilidad de enfermar, sino que reorienta la psicología hacia la promoción de la salud en las personas y, básicamente, pretende estudiar cuáles son los factores por los cuales un sujeto, a pesar de vivir circunstancias adversas, logra una calidad de vida satisfactoria. Plantea que la piedra angular es dilucidar cómo las personas controlan la tensión que genera la vida cotidiana, haciendo hincapié en que el estrés no necesariamente tiene un efecto negativo, sino que puede generar una adaptación activa al medio ambiente, a través de los recursos generalizados de resistencia (GRRs). Estos son recursos potenciales efectivos para resolver una amplia gama de problemas, promover un afrontamiento exitoso y hacer frente a estímulos perturbadores. Dichos estímulos ponen a prueba repetidamente al sujeto, generando a través del tiempo un sentido de coherencia (SOC). Con este concepto, Antonovsky hace referencia a la orientación global del individuo a partir de la cual el sujeto vivencia los estímulos como estructurados y predecibles, al mismo tiempo que se percibe con recursos para salir al encuentro de esos estímulos (Contini de González, 2001).

En general, todos estos desarrollos desde Maslow se proponen comprender las fortalezas y recursos personales que favorecen un mayor potencial de salud en las personas, y que explican las diferencias individuales a partir de las cuales hay personas que logran afrontar constructivamente las adversidades y alcanzar una calidad de vida satisfactoria (Contini de González, 2001; Gancedo, 2008). No pretende reemplazar el modelo médico patológico, sino complementarlo, al comprender a las personas como seres complejos que requieren un abordaje desde diferentes perspectivas, en donde el foco no esté en la prevención de la enfermedad, sino en la promoción de la salud desde el inicio de la vida y en los distintos ámbitos de socialización (familia, escuela, grupo de pares, trabajo), desarrollando así recursos y fortalezas personales.

A finales de la década de los '90, Martin Seligman introdujo la llamada psicología positiva durante su discurso inaugural como presidente de la American Psychological Association en Estados Unidos (Seligman \& Csikszentmilhalyi, 2000). Consiste en una serie de desarrollos que tienen por objetivo aportar nuevos conocimientos acerca del psiquismo, enfatizando los aspectos positivos de la experiencia, las fortalezas, virtudes y emociones positivas del ser humano. A continuación se presenta una descripción más detallada de la misma. 


\section{Psicología positiva}

La psicología positiva se define como el estudio científico de las experiencias positivas, los rasgos individuales positivos, las instituciones que facilitan su desarrollo y los programas que ayudan a mejorar la calidad de vida de los individuos, mientras previene o reduce la incidencia de la psicopatología y el malestar (Seligman 2003; Seligman \& Csikszentmihalyi, 2000). Es un campo que concierne al bienestar y al funcionamiento óptimo, y su objetivo es ampliar el foco de la psicología clínica más allá del sufrimiento y su consecuente alivio. Es definida también como el estudio científico de las fortalezas y virtudes humanas, tanto personales como cívicas e institucionales que guían a los individuos a tomar responsabilidades sobre su comunidad (Seligman \& Csikszentmihalyi, 2000). Castro Solano y LupanoPerugini (2013) señalan claramente cómo este modelo no solo pretende mejorar "lo que está mal", sino encontrar las fortalezas y virtudes de las personas para lograr una mejor calidad de vida, un mayor bienestar, la vida plena.

Este modelo, al igual que sus antecedentes, parte de concebir al individuo como sujeto activo, fuerte, con agencia, constructor de su propia realidad y con una importante capacidad de resistencia ante las adversidades de la vida (Mesurado, 2010). Es una visión optimista del ser humano que, siguiendo a Vilanova (1993), se reflejaba en todo el pensamiento de Maslow y Allport y que se puede rastrear hasta la obra Emilio o De la Educación de Rousseau (1762).

Seligman (2003) propone para la psicología positiva tres grandes pilares que, a su vez, constituyen las áreas de investigación centrales: a) el estudio de las emociones positivas -esperanza, alegría, confianza-, en tanto son esenciales en los momentos difíciles de la vida y permiten optimizar la salud y el bienestar humano; b) el estudio de la personalidad positiva, enfatizando las fortalezas y virtudes personales, como el valor, la integridad y la lealtad; y c) el estudio de las instituciones positivas, como la familia y la democracia, apuntando a una sociología positiva que tiene por objetivo que las comunidades prosperen y mejoren el desarrollo de las fortalezas y virtudes personales. Particularmente sobre las primeras dos áreas existe un cúmulo importante de desarrollos. Incluso se ha publicado un manual de virtudes y fortalezas que pretende ser la base para un Manual de Sanidades que sea la contrapartida de los manuales diagnósticos de trastornos mentales como el DSM (Casullo, 2008). Algunos de los tópicos centrales estudiados son el optimismo, la felicidad, la alegría y la esperanza; la creatividad y la experiencia de flow; crecimiento postraumático, la vida plena y el bienestar; el altruismo, los valores, la empatía y la capacidad de perdón, por mencionar solamente algunos (e.g., Casullo, 2005; Csikszentmihalyi, 1998; Donaldson, Dollweta \& Raoa, 2015; Fredrickson, 2001; Grinhauz \& CastroSolano, 2014; Mesurado, Richaud \& Niño, 2015; Richaud, 2014; Ryan \& Deci, 2001; 
Steca, Bassi, Caprara \& Fave, 2011; Tedeschi, Calhoun \& Groleau, 2015; Vázquez \& Hervás, 2008; Vitterso, 2003).

Las aplicaciones de la Psicología Positiva se extienden a diversos campos y no se restringen a nivel clínico individual, sino que incluyen el nivel grupal, organizacional y social (Castro-Solano \& Lupano-Perugini, 2013; Tarragona, 2013; Vázquez \& Hervás, 2008). Entre ellas se encuentran aproximaciones psicoterapéuticas que enfatizan lo positivo; intervenciones psicosociales y educacionales que promuevan la motivación, la creatividad y la promoción de recursos; y mejoras en el ciclo vital laboral y en el funcionamiento organizacional, como la gestión positiva de recursos humanos.

Si bien varias de las ideas, premisas teóricas y conceptos que propone la psicología positiva se pueden encontrar en desarrollos psicológicos previos (incluso el nombre mismo del modelo, dado que fue utilizado por primera vez por Maslow en 1954), e incluso en otros campos disciplinares como la filosofía y la ética, Gancedo (2008) destaca que uno de los méritos de la psicología de Seligman es haber funcionado como una suerte de catalizador del enfoque salugénico, que hasta ese momento seguía a la sombra del modelo patológico. La autora plantea que se ha convertido en un marco común al servir como una especie de "paraguas" bajo el cual convergen distintos desarrollos teóricos e investigaciones, y de este modo se han empezado a sistematizar los conocimientos que se tenían, con énfasis en el rigor científico.

\section{Abordaje de los comportamientos suicidas desde la Psicología Positiva}

Desde que la psicología se ha ocupado del estudio de los comportamientos suicidas se ha planteado una pregunta fundamental: ¿qué hace a la diferencia entre aquellas personas que recurren al suicidio como un modo de resolución del sufrimiento y los que nunca lo considerarían como una posibilidad? Para responder este interrogante se han estudiado ampliamente los llamados factores de riesgo, es decir aquellos que aumentan o favorecen la aparición de comportamientos suicidas. Sin embargo, a pesar de los importantes hallazgos, los autores representativos en el tema cada vez sostienen con mayor énfasis que es necesario investigar aquellos factores que resultan protectores al potenciar un funcionamiento saludable y adaptativo, a la vez que amortiguan el desarrollo de suicidalidad.

Es así que en las últimos años, la resiliencia y los factores protectores han cobrado particular relevancia en el estudio de los comportamientos suicidas (e.g., Johnson, Wood, Gooding, Taylor \& Tarrier, 2011; Osman et al., 2004; Rutter, Freedenthal \& Osman, 2008; Taliaferro \& Muehlenkamp, 2014), debido a que 
permiten dar cuenta de las diferencias individuales en la capacidad protectora frente a los factores de riesgo que, de otro modo, aumentarían el potencial suicida. Estos desarrollos conciben a la resiliencia no como un estado o resultado, sino como un concepto de orden superior o "constructo paraguas" (Hjemdal, 2007; Johnson, Gooding, Wood \& Tarrier, 2010; Osman et al., 2004) que incluye aquellos factores que contribuyen a un afrontamiento adaptativo, a pesar de experiencias con estresores que conllevan un alto riesgo de desarrollar patología.

Johnson et al. (2011) hablan de Resiliencia ante el Suicidio [Resilience to Suicide], definida en sentido amplio como una habilidad percibida, recursos o competencias que actúan como amortiguadores frente al impacto perjudicial del estrés y los factores de riesgo, disminuyendo la probabilidad de aparición de comportamientos suicidas. Esto constituye la llamada hipótesis de amortiguación [buffering hypothesis] que establece que más que un efecto directo, la resiliencia actuaría como una variable moderadora que atenúa el impacto de los factores de riesgo (Galarza, Martínez Festorazzi, Castañeiras \& Posada, 2014). Johnson et al. (2011) realizaron un relevamiento sistemático de las diferentes definiciones conceptuales y hallazgos empíricos respecto de cuáles son los llamados factores resilientes ante la suicidalidad, y destacan como factores resilientes el selfappraisal [autoevaluación] positivo, la regulación emocional, la capacidad para resolver problemas y el apoyo social.

Respecto de los aportes específicos de la psicología positiva a la comprensión y abordaje del comportamiento suicida, poco se ha encontrado en la literatura científica especializada. Cabe destacar el capítulo de libro publicado por Wingate et al. (2008) "Suicidio y cogniciones positivas: Psicología positiva aplicada al entendimiento y tratamiento del comportamiento suicida”. Los autores plantean que, desde la psicología positiva, el acento se pone en estudiar tres grupos de personas: a) aquellas que presentan múltiples factores suicidogénicos y se hallan en alto riesgo, pero que, a pesar de ello, no consideran al suicidio como un modo de resolución; b) quienes lo consideran, pero que no lo intentan; y c) aquellas personas que intentan quitarse la vida, pero no completan el acto. Sostienen que se deben indagar las estrategias que utilizan las personas para superar la desesperanza y la desesperación. Deben indagarse los recursos con los que cuentan las personas que efectivamente dicen que nunca se suicidarían, ya que podrían ser amortiguadores contra la conducta suicida. Esto último recuerda a lo planteado por Marsha Linehan (Linehan, Goodstein, Nielsen \& Chiles, 1983) cuando habla de razones para vivir, e incluso puede rastrearse una idea similar en un escrito de John B. Watson (1925/2014), “¿Por qué no me suicido?” [Why I don't commit suicide], recientemente publicado en la Revista de Historia de la Psicología. En dicho artículo, el conductista, preocupado por el aumento de la tasa de suicidios 
en jóvenes estadounidenses de aquel momento, intenta dar una explicación (y tratamiento) fuertemente ambientalista de la misma. Envió 100 cartas a diferentes personas solicitándoles que le comuniquen por qué no se suicidarían y expuso los resultados obtenidos. De alguna manera, esto podría considerarse un prototipo de investigación de factores protectores y razones para vivir.

En Wingate et al. (2008), los autores exponen tres aportes prometedores de la psicología positiva al abordaje de comportamientos suicidas. El primero refiere a comprender el rol del optimismo en el modo suicida, desde la teoría del optimismo de Seligman entendido en términos de atribución (no de disposición personal). A pesar de que escasean los estudios específicos, los autores han tomado como punto de partida el vasto cúmulo de investigación que existe sobre desesperanza (equivalente a pesimismo desde la teoría de Seligman) y suicidalidad (e.g., Abramson et al., 2000; Beck, Kovacs \& Weissman, 1975; Beck, Weisman, Lester \& Trexler, 1974; Daniel \& Goldston, 2012; Gooding et al., 2015; Later, Patsiokas, Clum \& Luscomb, 1979; O'Connor, Fraser, White, MacHale \& Masterton, 2008). Dado que la desesperanza es ampliamente considerada una de los principales predictores de suicidalidad en poblaciones clínicas, Wingate et al. infieren que el optimismo debe ser particularmente relevante para el estudio y tratamiento de los comportamientos suicidas; e incluso sostienen que es probable que sea un amortiguador importante contra la misma. Algunos estudios que han comenzado a desarrollar esta línea son el trabajo de O'Keefe \& Wingate (2013) y Davidson, Wingate, Rasmussen \& Slish (2009).

El segundo de los aportes que exponen lo reviste la teoría de las emociones positivas de Fredrickson (2001). Wingate et al. (2008) sostienen que las emociones positivas pueden tener un papel clave en el tratamiento y prevención de los comportamientos suicidas. Los autores sustentan esta premisa en la teoría de ampliación y construcción de emociones positivas, que propone que la experiencia de emociones positivas promueve la ampliación cognitiva y conductual, lo que le permite al sujeto concebir un repertorio más amplio de ideas y acciones de lo que es capaz cuando se encuentra experimentando un estado emocional negativo. Sumado a esto, la teoría de Fredrickson sostiene que en los momentos de ampliación del repertorio de pensamiento-acción se ve facilitada la posibilidad de desarrollar y acumular nuevos recursos y herramientas para resolver problemas. Estas presunciones de los autores son de particular interés cuando se considera que la llamada visión de túnel, el pensamiento constreñido o rigidez cognitiva, y las dificultades en el afrontamiento y resolución de problemas, son algunas de las principales características del modo cognitivo suicida, de manera que se estarían abordando algunos de los aspectos clave.

El tercer aporte que mencionan es la teoría de la autodeterminación de Deci 
y Ryan (2008), en combinación con la teoría interpersonal del suicidio de Joiner (2005), particularmente relevante para el tratamiento psicoterapéutico de los pacientes suicidas. La teoría de Deci y Ryan de la autodeterminación (2008) es una macroteoría de la motivación humana que permite entender el papel que juega la satisfacción de las necesidades psicológicas en el sentimiento de bienestar que desarrollan las personas. Se proponen tres necesidades psicológicas básicas: la autonomía, entendida como la dirección efectiva del transcurso de la propia vida; la necesidad de lazos afectivos, que refiere a la necesidad fundamental que tiene el ser humano de establecer conexiones interpersonales; y la competencia, que alude al empuje de las personas para dominar nuevas habilidades y situaciones. Por otro lado, la teoría interpersonal del suicido de Joiner y su equipo (Joiner, 2005; Van Orden et al., 2011) destaca como conceptos fundamentales del modelo la integración social [social connectedness], el sentido de pertenencia [belongingness], y el sentimiento de sobrecarga para los seres queridos [burdensomeness]. Según Joiner (2005), el deseo de la muerte está compuesto por dos estados psicológicos, la percepción de ser una carga para los demás y el sentimiento de no pertenecer a nada. Por sí mismos ninguno de estos dos estados son suficientes para despertar el deseo de la propia muerte, pero juntos dan lugar a un estado psíquico que puede ser mortal. Hay consenso indiscutido en el campo de la suicidología respecto al papel crucial que juegan la baja autoestima, un pobre sentido de autoeficacia, competencia y autonomía, y los sentimientos de aislamiento social y alienación, sobre todo en adolescentes (e.g., Daniel \& Goldston, 2012; King \& Merchant, 2008; Kleinman, Riskind \& Schaefer, 2014; Sharaf, Thompson \& Walsh, 2009). Recientemente, Tucker y Wingate (2014) han conducido un estudio empírico que ofrece evidencia con respecto al efecto de la satisfacción de las necesidades básicas como variable mediadora entre el sentimiento de no pertenencia y la percepción de sobrecarga, y el desarrollo de ideaciones suicidas. Según los autores, estos resultados sugieren que la satisfacción de necesidades psicológicas básicas en un factor predictor distal de las ideaciones suicidas, pero un predictor proximal de los factores de riesgos interpersonales propuestos por Joiner. De esta manera, si más estudios sostienen esta hipótesis, sería importante que el psicólogo clínico incluyera la evaluación del grado de satisfacción de estas necesidades psicológicas, ya que permitiría identificar potencialmente aquellos casos que se encontrarían más próximos a desarrollar factores de riesgo asociados a la suicidalidad. Asimismo, al poner de relieve conceptos como la necesidad de autonomía -que usualmente es ignorada en este tipo de pacientes-, la necesidad de vínculos y la motivación intrínseca/extrínseca, la aplicación de estas teorías en la práctica clínica puede ayudar al terapeuta a favorecer la adherencia al tratamiento (que suele ser un 
desafío importante con estos pacientes), y a la vez posibilitar un cambio real de la conducta suicida (para profundizar véase Wingate et al., 2008).

Por último, en el terreno de la evaluación de la suicidalidad, también se observa esta tendencia, cada vez mayor, a incluir los factores protectores como dimensiones clave a ser consideradas. Históricamente, y en correspondencia con lo dicho anteriormente, con excepción del Inventario de Razones para Vivir de Linehan et al. (1983; versión para adolescentes Osman, Downs \& Kopper, 1998), en general la mayoría de las técnicas diseñadas se han centrado en la evaluación de los factores de riesgo (Roaten, 2008; Rutter et al., 2008). Este cambio de enfoque probablemente se debe a dos razones: por un lado a la consolidación del modelo salugénico, y por otro a la evidencia empírica efectiva que sustenta el efecto moderador que muchos de los factores protectores o resilientes ejercen al disminuir la probabilidad de ocurrencia de la suicidalidad. Algunos de los más estudiados son autoestima (e.g., Creemers, Scholte, Engels, Prinstein \& Wiers, 2012; Hawton et al., 2012), apoyo social percibido y sentimiento de pertenencia (e.g. Kleinman et al., 2014; Van Orden et al., 2010), resolución de problemas (e.g., Linda, Marroquín \& Miranda, 2012; Quiñones, Jurska, Fener \& Miranda, 2015) y habilidades emocionales (e.g., Anestis, Bagge, Tull \& Joiner, 2011; Cha \& Nock, 2009), entre otros. Los autores representativos de la evaluación en suicidología concuerdan en que la clave está en analizar la interacción entre los factores protectores y los factores de riesgo que presenta una persona. Ejemplo de esto son las técnicas construidas por Osman y su equipo: el Inventario de Ideación Suicida Positiva y Negativa PANSI (Osman, Gutiérrez, Kopper, Barrios \& Chiros, 1998) y la Escala de Resiliencia ante el Suicidio SRI-25 (Osman et al., 2004), que miden factores que ayudan a preservar al sujeto frente al posible desarrollo de pensamientos y comportamientos suicidas. Estas técnicas han sido validadas en Colombia con adolescentes y jóvenes adultos (Villalobos-Galvis, 2010; Villalobos-Galvis, Árevalo Ojeda \& Rojas Rivera, 2012). Otro ejemplo es el Inventario de Riesgo Suicida para adolescentes IRIS de Hernández y Lucio (2003), un instrumento de screening para la detección y categorización del grado de riesgo en función del efecto moderador de los factores protectores. En la misma línea, en Argentina, Galarza, Castañeiras \& Fernández Liporace (2014) se encuentran abocadas al desarrollo de una escala de medición de suicidalidad en adolescentes que intenta mensurar el interjuego entre factores protectores y de riesgo. 


\section{Conclusiones}

Si bien aún existen pocos estudios que analicen empíricamente el impacto que los aportes de la psicología positiva pueden tener en el abordaje de los comportamientos suicidas, las presunciones teóricas y los datos preliminares resultan de interés. Particularmente prometedoras se estiman las aplicaciones de la teoría de la ampliación y construcción de las emociones positivas y la teoría de Joiner en combinación con la teoría de la autodeterminación al tratamiento de pacientes suicidas, dado que abordarían directamente aspectos clave del pensamiento suicida.

Se hace evidente una importante área de vacancia. Es esencial que se realicen investigaciones que se propongan indagar la relación entre suicidalidad y constructos positivos como optimismo, efectividad y pertenencia, por nombrar algunos, tanto para la comprensión y conceptualización como para la evaluación y el tratamiento. Sería interesante que se analice, por ejemplo, el impacto que podría tener incluir experiencias de flow y tareas que impliquen creatividad en el tratamiento con personas suicidas. La involucración en estas experiencias promueve una disminución de la conciencia de sí mismo y el tiempo, favorece la sensación de dominio y sentimientos de satisfacción (Mesurado, 2010; Tarragona, 2013). Esto sería altamente favorable para este tipo de pacientes, dado que son individuos cuya percepción de dominio sobre sí mismo y el entorno se encuentra seriamente disminuida: en general han perdido la capacidad de disfrute, presentan sentimientos de minusvalía e inutilidad, rigidez cognitiva, y escasos recursos para afrontar y resolver problemas.

En síntesis, las aplicaciones de la psicología positiva para el abordaje de la suicidalidad constituyen un área de investigación de vacancia que puede ofrecer alternativas novedosas, interesantes y enriquecedoras para el tratamiento de los comportamientos suicidas. Considerar los factores protectores, positivos, permitiría lograr una comprensión más cabal e integrada de los comportamientos suicidas y de las diferencias entre las subpoblaciones suicidas, lograr mayor precisión en la evaluación y estimación del potencial de suicidio, y mejorar las intervenciones terapéuticas.

i Para un análisis histórico exhaustivo se recomienda la lectura de Carpintero, H.; Mayor L. \& Zalbidea, M. A. (1990). Condiciones de surgimiento y desarrollo de la Psicología Humanista. Revista de Filosofía, 3(3), 71-82.

ii En este trabajo se tomarán los conceptos suicidalidad y comportamientos suicidas como sinónimos, en tanto pretenden aludir a todos los comportamientos incluidos en el espectro suicida sin resultado fatal -ideaciones suicidas, autolesiones, comportamientos suicidas, tentativas de suicidio- (Gvion \& Apter, 2012; Van Orden et al., 2010; Crosby, Ortega \& Melanson, 2011). 


\section{Referencias}

Allport, G. W. (1937/1965). Psicología de la personalidad. Buenos Aires: Paidós.

Anestis, M., Bagge, C. L., Tull, M. T., \& Joiner, T. E. (2011). Clarifying the role of emotion dysregulation in the interpersonal-psychological theory of suicidal behavior in an undergraduate sample. Journal of Psychiatric Research, 45(5), 603-611. doi:10.1016/j.jpsychires.2010.10.013

Antonovsky, A. (1988). Unraveling the mystery of health. How people manage stress and stay well. San Francisco: Jossey-Bass Publishers.

Bandura, A. (1977). Self-efficacy: Toward a unifying theory of behavioral change. Psychological Review, 84, 191-215.

Beck, A., Weisman, A., Lester, D., \& Trexler, L. (1974). The measurement of pessimism: the hopelessness scale. Journal of Counsulting and Clinical Psychology, 42, 861-865.

Buhgental, J. (1964). The third force in psychology. Journal of Humanistic Psychology, 4, 19-26.

Castro-Solano, A., \& Lupano-Perugini, M. L. (2013). The latin-american view of positive psychology. Journal of Behavior, Health \& Social Issues, 5(2), 15-31. doi: $10.5460 /$ jbhsi.v5.2.42250

Casullo, M. M. (2005). La capacidad para perdonar desde una perspectiva psicológica. Revista de Psicología de la PUCP, 23(1), 39-64.

Casullo, M. M. (2008). Prácticas en Psicología Positiva. Buenos Aires: Lugar Editorial.

Cha, C. \& Nock, M. K. (2009). Emotional intelligence is a protective factor for suicidal behavior. Journal of the American Academy of Child and Adolescent Psychiatry, 48(4), 422-430. doi: 10.1097/CHI.0b013e3181984f44

Contini de González, E. N. (2001). Hacia un cambio de paradigma: De la psicopatología al bienestar psicológico. Acta psiquiátrica y Psicológica de América Latina, 47(4), 306-315.

Creemers, D., Scholte, R., Engels, R., Prinstein, M., \& Wiers, R. (2012). Implicit and explicit self-esteem as concurrent predictors of suicidal ideation, depressive symptoms, and loneliness. Journal of Behavior Therapy and 
Experimental Psychiatry, 43(1), 638-646. doi: 10.1016/j.jbtep.2011.09.006

Crosby, A. E., Ortega, L., \& Melanson, C. (2011). Self-directed Violence Surveillance: Uniform Definitions and Recommended Data Elements, Version 1.0. Atlanta (GA), USA: Centers for Disease Control and Prevention, National Center for Injury Prevention and Control.

Csikszentmihalyi, M.(1998). Creatividad. El fluiry la psicología del descubrimiento y la invención. Barcelona: Paidós.

Daniel, S. S., \& Goldston, D. B. (2012). Hopelessness and Lack of Connectedness to Others as Risk Factors for Suicidal Behavior Across the Lifespan: Implications for Cognitive-Behavioral Treatment. Cognitive and Behavioral Practice, 19, 288-300. doi: 10.1016/j.cbpra.2011.05.003

Davidson, C. L., Wingate, L. R., Rasmussen, K. A., \& Slish, M. L. (2009). Hope as a predictor of interpersonal suicide risk. Suicide \& Life-Threatening Behavior, 39(5), 499-507. doi: 10.1521/suli.2009.39.5.499.

Deci, E. L., \& Ryan, R. M. (2008). Self-determination theory: A macrotheory of human motivation, development, and health. Canadian Psychology/ Psychologie canadienne, 49, 182-185. doi:10.1037=a0012801

Donaldson, S. I., Dollwet, M., \& Rao, M. A. (2015). Happiness, excellence, and optimal human functioning revisited: Examining the peer-reviewed literature linked to positive psychology. The Journal of Positive Psychology: Dedicated to furthering research and promoting good practice, 10(3), 185-195.

Fredrickson, B. (2001). The role of positive emotions in positive psychology. American Psychologist, 56(3), 218-226.

Galarza, A. L., Castañeiras, C. E., \& Fernández Liporace, M. (2014). Construcción y estudio de calidad psicométrica de la Escala Argentina de Suicidalidad para Adolescentes (EASA). Anuario de Proyectos e Informes de Becarios de Investigación de la Facultad de Psicología de la UNMDP, 11, 1009-1016.

Galarza, A. L., Martínez Festorazzi, V. S., Castañeiras, C. E., \& Posada, M. C. (2014). Riesgo suicida y resiliencia en un estudio con adolescentes argentinos. Acta Psiquiátrica y Psicológica de América Latina, 60(2), 98-108.

Gancedo, M. (2008). Historia de la Psicología Positiva. Antecedentes, aportes y proyecciones. En Casullo, M. M. (Ed.), Prácticas en Psicología Positiva. Buenos Aires: Lugar Editorial. 
Grinhauz, S. \& Castro-Solano, A. (2014). La evaluación de las virtudes y fortalezas del carácter en niños argentinos Summa Psicologica, 11, 115-126.

Gooding, P., Tarrier, N., Dunn, G., Shaw, J., Awenat, Y., Ulpha, F., \& Pratt, D., (2015). Effect of hopelessness on the links between psychiatric symptoms and suicidality in a vulnerable population at risk of suicide. Psychiatry Research, 230(2), 464-471. doi: 10.1016/j.psychres.2015.09.037

Gvion, Y. \& Apter, A. (2012). Suicide and suicidal behavior. Public Health Reviews, 34(2), 1-20

Hawton, K., Saunders, K., \& O'Connor, R. (2012). Self-harm and suicide in adolescents. Lancet, 379, 2373-2382.

Hernández-Cervantes, Q. \& Lucio, E. (2003). Inventario de riesgo suicida para adolescentes - IRIS. Versión para investigación. México: UNAM, Facultad de Psicología.

Hjemdal, O. (2007). Measuring protective factors: the development of two resilience scale in Norway. Child and Adolescence Psychiatric Clinics of North America, 16, 303-321.

Johnson, J., Gooding, P. A., Wood, A. M. \& Tarrier. N. (2010). Resilience as positive coping appraisals: Testing the schematic appraisals model of suicide (SAMS). Behaviour Research and Therapy, 48(3), 179-186. doi:10.1016/j. brat.2009.10.007

Johnson, J., Wood, A. M., Gooding, P., Taylor, P. \& Tarrier, N. (2011). Resilience to suicidality: The buffering hypothesis. Clinical Psychology Review, 31, 563591. doi: $10.1016 /$ j.cpr.2010.12.007

Joiner, T. E. (2005). Why people die by suicide. Cambridge, MA: Harvard University Press.

King, C. A. \& Merchant, C. R. (2008). Social and interpersonal factors relating to adolescent suicidality: A review of the literature. Archives of Suicide Research, 12(3), 181-196. doi: 10.1080/13811110802101203

Kleinman, E. M., Riskind, J. H. \& Schaefer, K. E. (2014). Social support and positive events as suicide resiliency factors: examination of synergistic buffering effects. Archives of Suicide Research, 18(2), 144-155. doi: 10.1080/13811118.2013.826155 
Linda, W. P., Marroquín, B., \& Miranda, R. (2012). Active and passive problem solving as moderators of the relation between negative life event stress and suicidal ideation among suicide attempters and non-attempters. Archives of Suicide Research, 16(3), 183-197. doi: 10.1080/13811118.2012.695233

Linehan, M., Goodstein, J., Nielsen, S. \& Chiles, J. (1983). Reasons for staying alive when you are thinking of killing yourself: The Reasons for Living Inventory. Journal of Consulting and Clinical Psychology, 51, 276-286.

Mariñelarena-Dondena, L., \& Gancedo, M. (2011). La Psicología Positiva: su primera década de desarrollo. diáLogos, 2(1), 67-77.

Maslow, A. (1954). Motivación y personalidad. Barcelona: Sagitario.

May, R. (1940). The Springs of Creative Living. United States: Whitmore \& Stone.

May, R. (1953). Man's Search for Himself. United States: Delta.

Mesurado, B. (2010). Hacia una conceptualización de la experiencia subjetiva de flow. Acta Psiquiátrica y Psicológica de América Latina, 56(1), 37-50.

Mesurado, B., Richaud, M. C. \& Niño, N. J. (2015). Engagement, Flow, Selfefficacy and Eustress of University Students: A cross-national comparison between the Philippines and Argentina. The Journal of Psychology: Interdisciplinary and Applied, 0(0), 1-24.

O'Connor, R. C., Fraser, L., White, M-C., MacHale, S. \& Masterton, G. (2008). A comparison of specific positive future expectancies and global hopelessness as predictors of suicidal ideation in a prospective study of repeat self-harmers. Journal of Affective Disorders, 110, 207-214. doi: 10.1016/j.jad.2008.01.008

O'Keefe V. M. \& Wingate, L. R. (2013). The role of hope and optimism in suicide risk for American Indians/Alaska Natives. Suicide \& Life-Threatening Behavior, 43(6), 621-33. doi: 10.1111/sltb.12044.

Osman, A, Gutierrez, P. M, Muehlenkamp, J. J., Dix-Richardson, F., Barrios, F. X. \& Kopper, B. A. (2004). Suicide Resilience Inventory-25: Development and preliminary psychometric properties. Psychological Reports, 94, 1349-1360.

Osman, A., Downs, W. R. \& Kopper, B. A. (1998). The Reasons for Living Inventory for Adolescents: development and psychometric properties. Journal of Clinical Psychology, 54(8):1063-1078.

Osman, A., Gutiérrez, P., Kopper, B., Barrios, F. \& Chiros, C. (1998). The 
Positive and Negative Suicide Ideation inventory: Development and validation. Psychological Reports, 82, 783-793.

Prada, E. C. (2005) Psicología Positiva y Emociones Positivas. Revista Electrónica Psicología Positiva.com. Disponible en http://www.psicologiapositiva.com/psicologiapos.pdf.pdf

Quiñones, V., Jurska, J., Fener, E. \& Miranda, R. (2015). Active and passive problem solving: moderating role in the relation between depressive symptoms and future suicidal ideation varies by suicide attempt history. Journal of Clinical Psychology, 71(4), 402-412. doi: 10.1002/jclp.22155

Richaud, M. C. (2014). Algunos aportes sobre la importancia de la empatía y la prosocialidad en el desarrollo humano. Revista Mexicana de Investigación en Psicología, 6, 171-176

Roaten, K. (2008). Cognition and suicide: the relationship between social problem-solving and suicidal behavior. Doctoral Thesis, University of Texas, EE.UU. Recuperado de http://repositories.tdl.org/utswmedr/bitstream/ handle/2152.5/594/roatenkimberly.pdf?sequence $=3$

Rogers, C. (1972). Psicoterapia centrada en el cliente. Buenos Aires: Paidós

Rousseau, J. J. (1762/2005). Emilio o De la Educación. Madrid: Alianza Editorial.

Rotter, J. B. (1966). Generalized expectancies of internal versus external control of reinforcement. Psychological Monographs, 80(1), 1-28.

Rutter, M. (1987). Psychosocial resilience and protective mechanisms. American Journal Orthopsychiatry, 57(3), 316-329.

Rutter, P. A., Freedenthal, S. \& Osman. A. (2008). Assessing protection from suicidal risk: Psychometric properties of the Suicide Resilience Inventory. Death Studies, 32, 142-153.

Ryan, R. M., \& Deci, E. D. (2001). On happiness and human potentials: A review of research on hedonic and eudaemonic well-being. Annual Review of Psychology, 52, 141-166.

Seligman, M. (2003). La auténtica felicidad. Buenos Aires: Vergara.

Seligman, M., \& Csikszentmilhalyi, M. (2000). Positive psychology. An introduction. American Psychologist, 55(1), 5-14. 
Sharaf, A. M., Thompson, E. A., \& Walsh, E. (2009). Protective Effects of Self-Esteem and Family Support on Suicide Risk Behaviors among At-Risk Adolescents. Journal of Child and Adolescent Psychiatric Nursing, 22(3), 160-168.

Steca, P., Bassi, M., Caprara, G. V., \& Fave, A. D. (2011). Parents' Self-efficacy Beliefs and Their Children's Psychosocial Adaptation During Adolescence. Journal of Youth Adolescence, 40(3), 320-331.

Taliaferro, L.A. \& Muehlenkamp, J. J. (2014). Risk and protective factors that distinguish adolescents who attempt suicide from those who only consider suicide in the past year. Suicide \& Life-Threatening Behavior, 44, 6-22. doi: 10.1111/sltb.12046

Tarragona, M. (2013). Psicología positiva y terapias constructivas: Una propuesta integradora. Terapia psicológica, 31(1), 115-125. doi: 10.4067/S071848082013000100011

Tedeschi, R. G., Calhoun, L. G. \& Groleau, J. M. (2015). Clinical Applications of Posttraumatic Growth. En S. Joseph (Ed.), Positive Psychology in Practice: Promoting Human Flourishing in Work, Health, Education, and Everyday Life, (2nd ed., pp. 503-518). New Jersey: John Wiley \& Sons, Inc.

Tucker, R. \& Wingate, L. R. (2014). Basic Need Satisfaction and Suicidal Ideation: A Self-Determination Perspective on Interpersonal Suicide Risk and Suicidal Thinking. Archives of Suicide Research, 18, 282-294. doi: 10.1080/13811118.2013.824839

Van Orden, A., Witte, T. K., Cukrowicz, K. C., Braithwaite, S., Selby, E. A. \& Joiner Jr., T. E. (2010). The interpersonal theory of suicide. Psychological Review, 117(2), 575-600. doi: 10.1037/a0018697.

Vázquez, C. \& Hervás, G. (2008). Psicología positiva aplicada. Madrid: Desclée de Brouwer.

Vilanova, A. (1993). Contribuciones a la Psicología Clínica. Algunos aportes teóricos de psicólogos notables. Buenos Aires: Ediciones ADIP.

Villalobos-Galvis, F. H. (2010). Validez y fiabilidad del inventario de Ideación Suicida Positiva y Negativa-PANSI en estudiantes colombianos. Universitas Psychologica, 9(2), 509-520.

Villalobos-Galvis, F. H., Árevalo Ojeda, C., \& Rojas Rivera, F. D. (2012). Adaptación del inventario de Resiliencia ante el Suicidio (SRI-25) en 
adolescentes y jóvenes de Colombia. Revista Panamericana de Salud Pública, $31(3), 233-239$.

Vitterso, J. (2003). Flow versus life satisfaction: A projective use of cartoons to illustrate the difference between the evaluation approach and the intrinsic motivation approach to subjective quality if life. Journal of Happiness Studies, 4, 141-167.

Watson, J. B. (1925/2014). Why I don’t commit suicide. Revista de Historia de la Psicología, 35(1), 37-46.

Wingate, L. R., Burns, A. .B, Gordon, K. H., Perez, M., Walker, R. L., Williams, F. M., \& Joiner, T. E. Jr. (2008). Suicidio y cogniciones positivas: Psicología positiva aplicada al entendimiento y tratamiento del comportamiento suicida. En T. E. Ellis (Ed.) Cognición y suicidio. Teoría, investigación y terapia (pp. 259-281). México: El Manual Moderno. 